\title{
Primary Small Cell Carcinoma of the Tongue
}

\author{
Naeem Latif ${ }^{1 *}$, Saba Imtiaz², Brad Wang', Fauzia Rana' and David Wolfson ${ }^{1}$
}

${ }^{1}$ Department of Medical Oncology and Pathology, University of Florida College of Medicine Jacksonville, Florida, USA

${ }^{2}$ Department of Medical Oncology, Shaukat Khanum Memorial Cancer Hospital and Research Centre, Lahore, Pakistan

\begin{abstract}
Primary small cell neuroendocrine carcinoma of the base of the tongue is a rare site for extra pulmonary small cell carcinomas. Histopathologically it is similar to small cell carcinoma of the lung with focal positivity for pancytokeratin and immune reactivity for the neuroendocrine markers.

Here we present a case of limited stage small cell neuroendocrine carcinoma of the base of the tongue in a middle aged smoker male for the first time in literature. We discuss its clinicopathological, radiological findings and management of extra pulmonary small cell carcinoma.
\end{abstract}

Keywords: Extra pulmonary; Small cell cancer; Tongue

\section{Introduction}

The vast majority of small cell carcinoma develops from the lung and only about $2.5 \%$ of small cell cancer arises in extra pulmonary sites [1]. The extra pulmonary small cell carcinoma is recognized as a clinicopathological entity distinct from small cell carcinoma of lung; however it is still often confused with metastatic small cell lung cancer [2]. The most commonly reported extra pulmonary sites with small cell carcinoma are aero-digestive tract including nasal cavity, para nasal sinuses, salivary glands, larynx, trachea and thyroid gland [3]. It has also been rarely found in breast, urinary bladder, prostate gland, ovaries and cervix. The extra pulmonary small cell lung cancer shares the same histopathological features as small cell lung cancer and is also chemotherapy sensitive. The Extra pulmonary small cell lung cancer is more aggressive and typically demonstrates a poorer prognosis [4].

\section{Case Report}

A 56 years old Caucasian male with a 20 pack year history of cigarette smoking and social alcohol drinker presented to the ENT clinic with left sided neck swelling for the past 2-3 months and it was progressively increasing in size. He also reported occasional difficulty in swallowing to solid food. There was no associated weight loss, his appetite was good and there was no pain. There were no other risk factors such as any history of chewing tobacco or betel nuts. The rest of systemic review was unremarkable. The patient's family history was significant for breast cancer in his mother and cancer of unknown primary in his father.

Examination of the oral cavity revealed a large growth at the base of tongue and also a left sided neck level II, $3.5 \mathrm{~cm}$ conglomerated firm mass was palpable. The rest of the systemic examination was unremarkable.

The fiber optic endoscopy by the ENT specialist showed a large proliferative growth arising from left base of the tongue, involving the vallecula, and epiglottis, while both of the vocal cords were normal and mobile.

The fine needle aspiration of the neck node revealed small rounded cells with scant cytoplasm that demonstrated nuclear molding. The immunohistochemical staining was positive for MAK-6 (dot like), CK-7, synaptophysin and chromogranin A, and it was negative for
LCA, p-63, CK-20 and cytokeratin. However, since primary small cell carcinoma of the tongue is rare, a recommendation for biopsy of the tongue lesion was made for correlation and definitive diagnosis (Figure $1)$.

The biopsy from the tongue lesion was consistent with small cell cancer and it was positive for $\mathrm{CK} A E 1 / 3$, and was negative for S-100. Hence a diagnosis of primary small cell carcinoma of the tongue with neck lymph node metastasis was confirmed.

The pretreatment PET/CT scan revealed a soft tissue mass at the base of the tongue extending inferiorly involving the left pharyngeal mucosal space and including a portion of the vallecula contiguous with the epiglottis. The mass was metabolically active with a maximum SUV of 7 , and a large metabolically active $3 \mathrm{~cm}$ lymph node was visualized posterior to the left internal jugular vein. Additional sub centimeter cervical lymph nodes were also noted at level II and a $1.5 \mathrm{~cm}$ lymph node was seen within the superior mediastinum without evidence of increased metabolic activity. Borderline prominent lymph nodes were also seen throughout the mediastinum, primarily the right para tracheal region, without evidence of increased metabolic activity. Sub segmental atelectasis of the bilateral lung bases was noted without a primary lung lesion. There was no other metastatic disease seen on PET/CT scan.

The case was discussed in the multi-disciplinary Head and Neck tumor conference and it was decided to treat this as a case of extra pulmonary small cell lung carcinoma primarily arising from the base of the tongue. There was a possibility that the tumor may have arisen from minor salivary gland under the tongue. The tumor was staged as limited (T2 N2b M0) stage disease, and it was recommended to treat him with concomitant chemotherapy and radiation.

*Corresponding author: Naeem Latif, MD, Assistant Professor of Medicine Department of Medical Oncology and Pathology, University of Florida, Jacksonville, 655 West 8th Street, 4N, Pavilion, Jacksonville, FL 32209, USA, Tel: 904-244-1680; Fax: 904-244-1681; E-mail: Naeem.latif@jax.ufl.edu

Received January 24, 2012; Accepted February 18, 2012; Published February 20, 2012

Citation: Latif N, Imtiaz S, Wang B, Rana F, Wolfson D (2012) Primary Small Cell Carcinoma of the Tongue. J Cancer Sci Ther S5:007. doi:10.4172/1948-5956.S5-007

Copyright: (c) 2012 Latif N, et al. This is an open-access article distributed under the terms of the Creative Commons Attribution License, which permits unrestricted use, distribution, and reproduction in any medium, provided the original author and source are credited. 


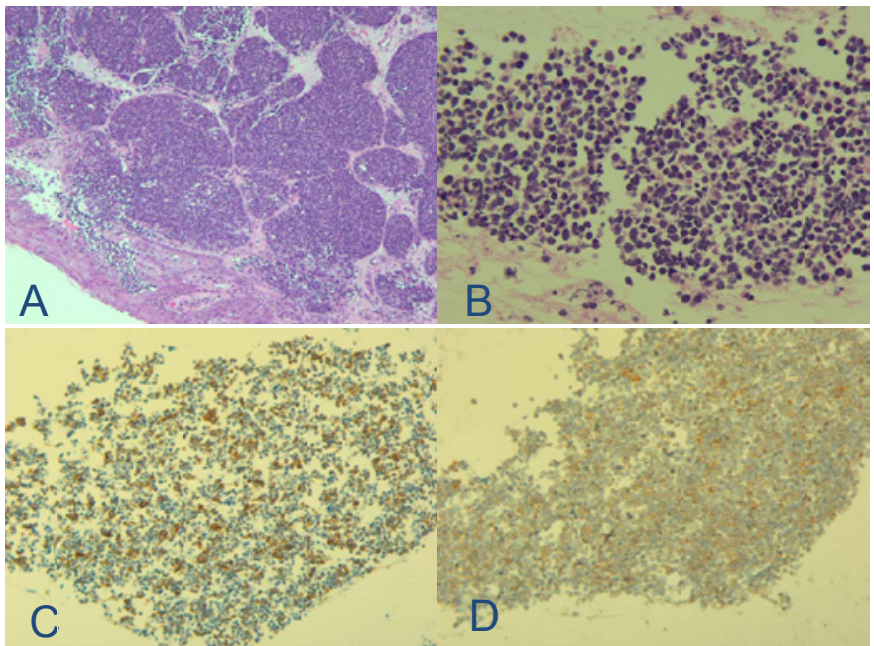

Figure 1: Representative sections of the small cell carcinoma. A) H \& E stained section of biopsy (100 $x$ magnifications) showing nests of small cell carcinoma infiltrating submucosa and squamous epithelium. B) Cell block (400 x magnifications) displaying aggregates of small cell carcinoma intermixed with inflammatory cells. C) Positive immunohistochemical (IHC) stain for pancytokeratin (MAK6) (200 x magnification). D) Positive IHC stain for synaptophysin (200 x magnifications). The tumors cells are also positive for chromogranin and TTF1, but negative for CD45, S100, p63 and CK20 (not shown).

Due to aggressive nature of extra pulmonary small cell carcinoma, we decided to treat him with full dose Cisplatin and Etoposide every twenty one days concomitant with radiation therapy. Patient completed 2 cycles of chemotherapy and total 74 Gy of radiation (both photons and electrons were used). During treatment patient developed severe mucositis and required PEG tube placement for nutrition. The follow up PET/CT scan after 2 cycles of chemotherapy and radiation shows complete resolution of the tongue and neck mass and there was minimal post radiation uptake, SUV was not recorded. Patient could not continue further chemotherapy due pancytopenia and severe mucositis. Then patient moved to another state with his family and lost to follow up.

\section{Discussion}

Cancers of the base of the tongue are relatively uncommon and typically occur in fifth through seventh decade of life; men are afflicted three to five times more often than women $[5,6]$. Like other head and neck cancers, tobacco, alcohol abuse and more recently human papillomavirus (HPV) infection represents the most significant risk factors for the development of tongue cancers [5]. Histologically, almost all oropharyngeal cancers are squamous cell carcinomas and very rarely any other histology is seen [6]. Primary small cell carcinoma of the tongue has never been reported in the literature to the best of our search, and here we take the opportunity to report this first case of extra pulmonary primary small cell tongue carcinoma.

Tumors metastasizing to tongue are relatively rare only $0.2-1.6 \%$ of all tongue cancers and the prognosis is poor because the primary tumor has already metastasized. The common cancers which metastasis to the tongue is lung, breast, skin, gastrointestinal tract and liver [2,7]. The base of the tongue is more common site for metastatic disease compared to the anterior tongue [8]. Rarely the tongue metastasis can be the initial presentation of an occult primary cancer as reported by Terashima et al. [2].

Small cell lung cancer is a high grade rapidly growing tumor with potential to metastasize early in the course of the disease. It commonly metastasizes to bones, brain, oral cavity, gingival, tongue, parotid gland, lymph nodes, liver and adrenal glands [7]. The extra pulmonary small cell cancer is now recognized as a separate clinicopathological entity but behaves similar to small cell lung cancer with more aggressive course and carries a poorer prognosis. It has more male preponderance and more common in smokers $[1,3]$.

Our patient did not have any respiratory symptoms and the history of tongue and neck mass was of short duration. At the time of diagnosis the disease has already spread to the neck lymph nodes, showing the aggressive nature of the disease. There was no parenchymal lung lesion on PET/CT scan suggesting non pulmonary origin of this cancer. The para tracheal and mediastinal lymph nodes noted on PET/CT were small and were metabolically not active. The bronchoscopic examination was not entertained because there was no obvious lung lesion seen on PET/CT scan

The histological criteria for the diagnosis of extra pulmonary small cell lung carcinoma are similar to that of small cell lung cancer. The pathological specimen from both the tongue lesion and the neck node showed cells with hyperchromatic nuclei, inconspicuous nucleoli, and scanty cytoplasm. The pancytokeratin (CK AE1/3 and MAK-6) showed focal positivity that is consistent with small cell carcinoma. The immunostains for melanoma, primary lung, breast, colon and lymphoma were negative. The cells were positive for neuroendocrine markers such as chromogranin A and synaptophysin on immunohistochemistry, similar to reported by Kim and Latif et al. $[3,4]$ for small cell cancer.

The small cell lung cancer and extra pulmonary small cell carcinoma have similar histopathological appearance and are mostly positive for neuroendocrine markers and it is difficult to localize the primary site of origin.

Small cell cancer is a systemic disease and localized treatment alone is not sufficient; therefore, even at an early stage, multimodality therapy including systemic therapy is preferred. Unlike the small cell lung cancer, the natural history of most cases of extra pulmonary small cell cancer remains elusive and therefore optimal therapy cannot be determined $[3,4]$. The clinical course of the tumor in patients with extra pulmonary small cell cancer is more aggressive and often recurrent. There are no standard guidelines to treat patients with extra pulmonary small cell cancer however; because of its relative chemo sensitive nature, the majority of extra pulmonary small cell cancer patients are treated with Platinum based chemotherapy regimens similar to small cell lung cancer $[3,4]$. Our patient was treated with Cisplatin and Etoposide chemotherapy concomitant with radiation to the tongue base and ispilateral neck with excellent response to treatment.

The median survival for extra pulmonary small cell cancer irrespective of site of origin is almost 14 months [3]. The initial stage at the time of diagnosis and location of the disease are independent prognostic factors for survival, and it is unfavorable when patient presents with extensive stage disease [3]. Small cell cancer of the gastro intestinal tract or oral cavity is rare and most commonly found in patients with advanced age and caries a dismal prognosis [9]. 
Citation: Latif N, Imtiaz S, Wang B, Rana F, Wolfson D (2012) Primary Small Cell Carcinoma of the Tongue. J Cancer Sci Ther S5:007. doi:10.4172/1948-5956.S5-007

\section{References}

1. Ledermann JA (1992) Extrapulmonary small cell carcinoma. Postgrad Med $J$ 68: 79-81.

2. Terashima T, Matsuzaki T, Kawada I, Nishida J, Tanaka Y, et al. (2004) Tongue metastasis as an initial presentation of a lung cancer. Intern Med 43: 727-730.

3. Kim KO, Lee HY, Chun SH, Shin SJ, Kim MK, et al. (2006) Clinical overview of extra pulmonary small cell carcinoma. J Korean Med Sci 21: 833-837.

4. Latif N, Rosa M, Samian L, Rana F (2010) An unusual case of primary small cell neuroendocrine carcinoma of the breast. Breast J 16: 647-651.

5. Hu KS, Harrison LB, Culliney B (2004) Cancer of the oropharynx. In: Harrison LB, Sessions RB, Hong WK (eds): Head and Neck Cancer: A Multidisciplinary Approach. (2nd edn), Philadelphia Pa: Lippincott Williams \& Wilkins: 306-351.
6. Licitra L, Bernier J, Grandi C, Merlano M, Bruzzi P, et al. (2002) Cancer of the oropharynx. Crit Rev Oncol Hematol 41: 107-122.

7. Yildiz O, Buyuktas D, Ekiz E, Selcukbiricik F, Papila I, et al. (2011) Facial nerve palsy: an unusual presenting feature of small cell lung cancer. Case Rep Oncol 4: $35-38$.

8. Kurt M, Bulut N, Aksoy S, Kosemehmetoglu K, Kars A (2006) Anterior tongue metastasis from lung cancer. South Med J 99: 784-785.

9. Medgyesy CD, Wolff RA, Putnam JB Jr, Ajani JA (2000) Small cell carcinoma of the esophagus: the University of Texas M. D. Anderson Cancer Center experience and literature review. Cancer 88: 262-267.
This article was originally published in a special issue, Cancer Research Clinical \& Experimental handled by Editor(s). Dr. Richard D. Finkelman, AstraZeneca LP Clinical, USA; Dr. Jimmy Thomas Efird, University of North Carolina, USA; Dr. Yanming Wang, Case Western Reserve University, USA 